\title{
CONSEQUENCES OF THE OSTROGRADSKY-GAUSS THEOREM FOR NUMERICAL SIMULATION IN AEROMECHANICS
}

\author{
Evelina Prozorova ${ }^{* 1}$ 伲 \\ ${ }^{* 1}$ St. Petersburg State University, University av. 28, Peterhof, 198504, Russia
}

DOI: https://doi.org/10.29121/granthaalayah.v8.i6.2020.549

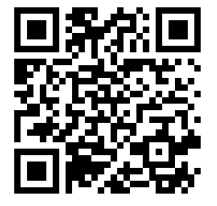

Article Type: Research Article

Article Citation: Evelina Prozorova. (2020). CONSEQUENCES OF THE OSTROGRADSKY-GAUSS THEOREM FOR NUMERICAL SIMULATION IN AEROMECHANICS. International Journal of Research GRANTHAALAYAH, 8(6), 270-275

https://doi.org/10.29121/granthaa layah.v8.i6.2020.549

Received Date: 12 May 2020

Accepted Date: 30 June 2020

Keywords:

Angular Momentum

Conservation Laws

Non-Symmetrical Stress Tensor

Conjugate Problem the Navie-Stokes

Separate Problem

\begin{abstract}
Using the Ostrogradsky-Gauss theorem to construct the laws of conservation and replacement of the integral over the surface by the integral over the volume, we neglect the integral term outside, i.e. neglect the circulation on the sides of the elementary volume (in the twodimensional case, this is clearly visible). Circulation means the presence of rotation, which in turn means the presence of a moment of force (angular momentum). As a result, we have a symmetric stress tensor, a symmetric velocity tensor, etc. Static pressure, as follows from kinetic theory, there is a zero-order quantity; the terms associated with dissipative effects are first-order quantities. It does not follow from the Boltzmann equation and from the phenomenological theory that the pressure in the Euler equation is equal to one third of the sum of the pressures on the corresponding coordinate axes. The inaccuracy of determining the velocities in the stress tensor in the stress tensor does not strongly affect the results at low speeds. All these issues are discussed in the work. As example in this paper suggests task of flowing liquid at little distance of two parallel plates.
\end{abstract}

\section{INTRODUCTION}

The aim of the work is to study the consequences of using the Ostrogradsky-Gauss theorem in continuum mechanics in deriving conservation laws and numerically solving received equations. Conservation laws were obtained experimentally and therefore were originally written in integral form. Differential laws are obtained in two ways: using the finite volume method for an elementary volume and using the Ostrogradsky Gauss theorem by replacing the surface integral to the volume integral, that is, taking the integral by parts with further use of the theorems on the conditions Integral turning in zero. Usually the derivation of conservation laws is analyzed using the Ostrogradsky-Gauss theorem for a fixed volume without moving. The theorem is a consequence of the application of the integration in parts at the spatial case. In reality, in mechanics and physics gas and liquid move and not only progressively, but also rotate. Discarding the term means ignoring the velocity circulation over the surface of the selected volume. When studying vortices, the whole theory is based on the action of the law of conservation of moments [1], [2] partially the moment is used when considering stresses in beams. When taking into account the motion of a gas, the extra-integral term is difficult to introduce into the differential equation. Therefore, to account

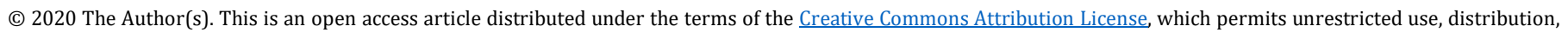
and reproduction in any medium, provided the original author and source are credited. 
for all components of the motion, it is proposed to use an integral formulation. The classical Boltzmann equation does not fulfill the law of conservation of momentum. From the definition of pressure, both from the classical Boltzmann equation and from the modified one, it does not follow that hydrostatic pressure is one third of the sum of the pressures at the coordinate surfaces. The equations of motion obtained from the Boltzmann equation correspond to the zero the order for the Euler equations and the first order for the Navier-Stokes equations. Hydrostatic pressure is a zero-order value, but the theory remains the same when determining different pressures at each surface, i.e. $p_{x}, p_{y}, p_{z}$ The use of one pressure is possible under equilibrium conditions (Pascal's law), but for nonequilibrium conditions the fact is not obvious. This is highlighted in the textbook [1]. Back in 1970, in the textbook it was said, "First of all, we note that along with the distribution of volume and surface forces, for the sake of generality, we should also consider the volume and surface distributions of pairs of forces (moments), on the possibility of which in continuous media is currently indicated ". In the theory of elasticity, when considering the relationship between the components of the strain tensor and the stress tensor, the experimental fact is used that the components $\sigma_{x}, \sigma_{y}, \sigma_{z}$ (stresses) normal to the side of elementary element are proportional to the sum of the stresses of the other components and they all differ. The introduced components are the result of the action of moments of force. The prevailing theory is connected with the fact that the derivation of conservation laws in the theory of elasticity excludes the contribution of the distributed moment to the equilibrium of forces. As a result, the law of equilibrium of forces and moments of forces are considered separately.

In solving specific problems, the contribution of the distributed moment is often studied, but the symmetry condition of the stress tensor remains. The existing classical theory is constructed so that the main role is played by pressure forces. This is probably why, at low speeds, results coincidences with experiment are obtained. However, calculations even for a potential flow lead to the formation of a vortex sheet. For Euler's equations, this means that Pascal's law in the non-equilibrium case does not work and it is necessary to consider separately not pressure $p$, but $p_{x}, p_{y}, p_{z}$. An example of the importance of separating the individual components is the study of the processes of wave propagation in a rotating fluid [3] with the introduction of a item related to rotation. in pressure gradient. Sometimes, when solving problems in the theory of elasticity, the first invariants are used, but this can be done if the tensor is symmetric. When considering the vortex motion, the tensor is asymmetric [4]. The equilibrium condition is fulfilled if there are no internal and external forces, however, any surface forces can be converted by changing variables into internal forces and deformations occur. When studying and writing down conservation laws, it is important to distinguish between equilibrium and no equilibrium cases.

At equilibrium in the mechanics of a continuous medium, equilibrium means the homogeneity of the distribution of all macro parameters. However, any additional external influence leads to the emergence of certain disturbances and creates a distributed moment, which creates additional force and gives asymmetric pressure values. Writing separately the law of equilibrium for forces and separately for the moments of forces without taking into account the mutual influence, and the moment creates additional force, we come to the conclusion about the symmetry of the stress tensor. In previous works, taking into account the angular momentum law nonsymmetrical stress tensor is received. The method for calculation of nonsymmetrical part was suggested. The equations for gas were found from the modified Boltzmann equation and the phenomenological theory. For a rigid body the equations were used of the phenomenological theory, but changed their interpretation. For rarefied gas the second term in collision integral of the Boltzmann equation is taken into account to calculate the self- diffusion and thermo-diffusion that was foretell by S. Wallander. The Hilbert paradox was solved. We discussed the problems that can be appearing for consideration the angular momentum variation in an elementary volume near the surface and into boundary layer. Conditions of the existence A.N. Kolmogorov inertia interval are established. Conjugated conditions at surface without the Knudsen layer are written to count friction and heat flow to the surface. Conditions of influence of angular momentum are discussed. The examples were given. The numerical examples of solving the simplest problems of the theory of elasticity, a boundary layer, and kinetic theory were given [5], [6], [7]. The inclusion of velocity circulation in potential flows means the inclusion of rotational velocity components.

The work discusses the listed issues and, if possible, gives answers to some of them.

\section{EQUATIONS}

One of the options for deriving symmetry conditions for pressure tensor: 
We have angular momentum

$\int_{\omega} \rho\left(x_{2} f_{3}-x_{3} f_{2}\right) d x=M_{x}$

However, moment creates additional force and the symmetry of the stress tensor is broken.

Taking into account the equilibrium equation, we obtain

$\int_{\omega}\left[x_{2}\left(\frac{\partial \sigma_{i 3}}{\partial x_{i}}+\rho f_{3}\right)+\sigma_{23}-x_{3}\left(\frac{\partial \sigma_{j 2}}{\partial x_{j}}+\rho f_{2}\right)-\sigma_{32}\right] d x=0$.

In classical case:

$$
\begin{gathered}
0=\rho f_{1}+\left(\frac{\partial \sigma_{x}}{\partial x}+\frac{\partial \tau_{x y}}{\partial y}+\frac{\partial \tau_{x z}}{\partial z}\right), \\
0=\rho f_{2}+\left(\frac{\partial \tau_{y x}}{\partial x}+\frac{\partial \sigma_{y}}{\partial y}+\frac{\partial \tau_{y z}}{\partial z}\right), \\
0=\rho f_{3}+\left(\frac{\partial \tau_{z x}}{\partial x}+\frac{\partial \tau_{z y}}{\partial y}+\frac{\partial \sigma_{z}}{\partial z}\right)
\end{gathered}
$$

In our case

$$
\begin{aligned}
& \rho\left(\frac{\partial u}{\partial t}+u \frac{\partial u}{\partial x}+v \frac{\partial u}{\partial y}+w \frac{\partial u}{\partial z}\right)=\rho f_{1}+\frac{\partial \sigma_{x x}}{\partial x}+\frac{\partial \sigma_{y x}}{\partial y}+\frac{\partial \sigma_{z x}}{\partial z}+\rho f_{M_{x}} \\
& \rho\left(\frac{\partial v}{\partial t}+u \frac{\partial v}{\partial x}+v \frac{\partial v}{\partial y}+w \frac{\partial v}{\partial z}\right)=\rho f_{2}+\frac{\partial \sigma_{x y}}{\partial x}+\frac{\partial \sigma_{y y}}{\partial y}+\frac{\partial \sigma_{z y}}{\partial z}+\rho f_{M_{y}} \\
& \rho\left(\frac{\partial w}{\partial t}+u \frac{\partial w}{\partial x}+v \frac{\partial w}{\partial y}+w \frac{\partial w}{\partial z}\right)=\rho f_{3}+\frac{\partial \sigma}{\partial x}+\frac{\partial P \sigma_{y z}}{\partial y}+\frac{\partial \sigma_{z z}}{\partial z}+\rho f_{M_{z}} \\
& y\left(\frac{\partial \sigma_{x z}}{\partial x}+\frac{\partial \sigma_{y z}}{\partial y}+\frac{\partial \sigma_{z z}}{\partial z}+\rho f_{3}\right)-z\left(\frac{\partial \sigma_{x y}}{\partial x}+\frac{\partial \sigma_{y y}}{\partial y}+\frac{\partial \sigma_{z y}}{\partial z}+\rho f_{2}\right)+\sigma_{z y}-\sigma_{z y}+M_{x}=0 \\
& x\left(\frac{\partial \sigma_{x y}}{\partial x}+\frac{\partial \sigma_{y y}}{\partial y}+\frac{\partial \sigma_{z y}}{\partial z}+\rho f_{2}\right)-y\left(\frac{\partial \sigma_{x x}}{\partial x}+\frac{\partial \sigma_{y x}}{\partial y}+\frac{\partial \sigma_{z x}}{\partial z}+\rho f_{1}\right)+\sigma_{y x}-\sigma_{x y}+M_{y}=0 \\
& x\left(\frac{\partial \sigma_{x z}}{\partial x}+\frac{\partial \sigma_{y z}}{\partial y}+\frac{\partial \sigma_{z z}}{\partial z}+\rho f_{1}\right)-z\left(\frac{\partial \sigma_{x x}}{\partial x}+\frac{\partial \sigma_{y x}}{\partial y}+\frac{\partial \sigma_{z x}}{\partial z}+\rho f_{2}\right)+\sigma_{z x}-\sigma_{x z}+M_{z}=0
\end{aligned}
$$

\section{THE INFLUENCE OF THE MOMENT IN THE PROBLEM OF FLUID MOTION BETWEEN TWO CLOSELY SPACED PARALLEL PLATES}

Consider the flow of a very viscous fluid between two parallel plates, the distance $h$ between which we will consider very small. Reynolds number is small, external forces are absent. Initial statement of the problem

$$
\begin{aligned}
& \frac{\partial p}{\partial x}=\mu\left(\frac{\partial^{2} v_{x}}{\partial x^{2}}+\frac{\partial^{2} v_{x}}{\partial y^{2}}+\frac{\partial^{2} v_{x}}{\partial z^{2}}\right), \\
& \frac{\partial p}{\partial y}=\mu\left(\frac{\partial^{2} v_{e}}{\partial x^{2}}+\frac{\partial^{2} v_{y}}{\partial y^{2}}+\frac{\partial^{2} v_{y}}{\partial z^{2}}\right) \\
& \frac{\partial p}{\partial z}=\mu\left(\frac{\partial^{2} z}{\partial x^{2}}+\frac{\partial^{2} v_{z}}{\partial y^{2}}+\frac{\partial^{2} u_{z}}{\partial z^{2}}\right)
\end{aligned}
$$


The axes $O x, O y$ lie in one of the boundary planes, the axis $\mathrm{Oz}$ is directed perpendicular to these planes and the equations of the boundary planes $\mathrm{z}=0, \mathrm{z}=\mathrm{h}$. It is assumed that the velocity is directed parallel to the boundary planes, so that

$$
v_{z}=0
$$

Due to the small distance between the plates, the order of the derivative $\frac{\partial v_{z}}{\partial x}$ is large compared to the derivatives $\frac{\partial v_{x}}{\partial x}$ and $\frac{\partial v_{x}}{\partial y}$. The order of the derivatives $\frac{\partial^{2} v_{x}}{\partial z^{2}}$ is large compared to the orders of the derivatives $\frac{\partial^{2} v_{x}}{\partial x^{2}}$ and $\frac{\partial^{2} v_{x}}{\partial y^{2}}$. Then the equations take the form.

$$
\frac{\partial p}{\partial x}=\mu \frac{\partial^{2} v_{x}}{\partial z^{2}}, \frac{\partial p}{\partial y}=\mu \frac{\partial^{2} v_{y}}{\partial z^{2}}, \quad \frac{\partial p}{\partial z}=0, \frac{\partial v_{x}}{\partial x}+\frac{\partial v_{\mathrm{H}}}{\partial \mathrm{H}}=0 .
$$

We show the influence of the moment in this problem.

We take into account that the Euler equations provide the main order. In classical theory the pressure is selected in the center of the cell and is the same for all coordinate axes and is equal to p. Suppose that the moment acts and creates a small additional force.

$\tilde{p}_{1}=p+\in f_{1}, \quad \tilde{p}_{2}=p+\in f_{2}, \quad$ The result of the additional force will be new velocity

$$
\tilde{v}_{x}=v_{x}+\in V_{1}, \quad \tilde{v}_{y}=v_{y}+\in V_{2}
$$

$\frac{\partial \tilde{p}_{1}}{\partial x}=\mu \frac{\partial^{2} \widetilde{v}_{x}}{\partial z^{2}}, \frac{\partial \tilde{p}_{2}}{\partial y}=\mu \frac{\partial^{2} \widetilde{v}_{x}}{\partial z^{2}}, \frac{\partial \tilde{p}_{1}}{\partial z}=0, \quad \frac{\partial \tilde{p}_{2}}{\partial z}=0, \quad \frac{\partial \tilde{v}_{x}}{\partial x}+\frac{\partial \tilde{v}_{y}}{\partial x}=0$.

$f_{1}=-y \frac{\partial \tilde{p}_{1}}{\partial x}, f_{2}=x \frac{\partial \tilde{p}_{2}}{\partial y}$.

$$
\begin{gathered}
\tilde{v}_{x}=\frac{z^{2}}{2} \frac{\partial \tilde{p}_{1}}{\partial x}+z A_{1}(x, y)+B_{1}(x, y) . \\
\tilde{v}_{y}=\frac{z^{2}}{2} \frac{\partial \tilde{p}_{1}}{\partial x}+z A_{2}(x, y)+B_{2}(x, y) .
\end{gathered}
$$

By the boundary conditions, we have

$$
V_{1}=-\frac{y}{2 \mu} \frac{\partial^{2} \tilde{p}_{1}}{\partial x^{2}} z(h-z), \quad V_{2}=-\frac{y}{2 \mu} \frac{\partial^{2} \tilde{p}_{1}}{\partial y^{2}} z(h-z),
$$

Under the assumption of a small influence of the moment

$V_{1}=-\frac{y}{2 \mu} \frac{\partial^{2} p}{\partial x^{2}} z(h-z), \quad V_{2}=-\frac{y}{2 \mu} \frac{\partial^{2} p}{\partial y^{2}} z(h-z)$.

We get two equations that pressure must satisfy

$$
\frac{\partial^{2} p}{\partial x^{2}}+\frac{\partial^{2} p}{\partial y^{2}}=0, \text { and } y \frac{\partial}{\partial x}\left(-\frac{\partial^{2} p}{\partial x^{2}}\right)+x \frac{\partial}{\partial y} \frac{\partial^{2} p}{\partial y^{2}}=0 .
$$

Therefore, the following equation must be satisfied

$$
y \frac{\partial}{\partial x}\left(\frac{\partial^{2} p}{\partial x^{2}}\right)-x \frac{\partial}{\partial y} \frac{\partial^{2} p}{\partial x^{2}}=0 .
$$

The results allow us to hope that the pressure distribution along the corresponding axes is responsible for large eddies. Dispersion create small waves. Viscosity is responsible for dissipation. 


\section{THE PROPOSED VERSION OF THE EQUATIONS}

We will use the expansion that is in the textbooks [], but we will make a decomposition with respect near the center of inertia of the elementary volume $r_{c}=r^{\prime}\left(x^{\prime}, y^{\prime}, z^{\prime}\right)$

$$
\left.\begin{array}{rl}
u^{\prime} & =u+\frac{\partial u}{\partial x}\left(x-x^{\prime}\right)+\frac{\partial u}{\partial y}\left(y-y^{\prime}\right)+\frac{\partial u}{\partial z}\left(z-z^{\prime}\right) \\
v^{\prime} & =v+\frac{\partial v}{\partial x}\left(x-x^{\prime}\right)+\frac{\partial v}{\partial y}\left(y-y^{\prime}\right)+\frac{\partial v}{\partial z}\left(z-z^{\prime}\right) \\
w^{\prime} & =w+\frac{\partial w}{\partial x}\left(x-x^{\prime}\right)+\frac{\partial w}{\partial y}\left(y-y^{\prime}\right)+\frac{\partial w}{\partial z}\left(z-z^{\prime}\right)
\end{array}\right\}
$$

We will not divide the speed into divergent and vortex parts. We leave Newton's law for the effect of viscosity. In modern computational mechanics, no difficulties will arise.

Then the viscous stress tensor $\dot{S}$ will have the form

$$
\dot{S}=\left[\begin{array}{lll}
\frac{\partial u}{\partial x} & \frac{\partial u}{\partial y} & \frac{\partial u}{\partial z} \\
\frac{\partial v}{\partial x} & \frac{\partial v}{\partial y} & \frac{\partial v}{\partial z} \\
\frac{\partial w}{\partial x} & \frac{\partial w}{\partial y} & \frac{\partial w}{\partial z}
\end{array}\right]
$$

For an incompressible fluid, the equations remain the same in the case for the non-compressible liquid without the angular momentum, the pressure gradient will change. Really,

$$
\begin{aligned}
& p_{x x}=p_{1}+\mu \frac{\partial p_{x x}}{\partial x}, \quad p_{y y}=p_{2}+\mu \frac{\partial p_{y y}}{\partial y}, \quad p_{z z}=p_{3}+\mu \frac{\partial p_{z z}}{\partial z} . \\
& p_{x y}=\mu \frac{\partial u}{\partial y}, \quad p_{y x}=\mu \frac{\partial v}{\partial x}, p_{y z}=\mu \frac{\partial v}{\partial z}, \quad p_{z y}=\mu \frac{\partial w}{\partial y}, \quad p_{z x}=\mu \frac{\partial w}{\partial x}, \\
& p_{x z}=\mu \frac{\partial u}{\partial z} . \\
& \frac{\partial p_{x x}}{\partial x}+\frac{\partial p_{y x}}{\partial y}+\frac{\partial p_{z x}}{\partial z}=-\frac{\partial p_{1}}{\partial x}+\mu \nabla^{2} u,
\end{aligned}
$$

for the remaining components is similar. It is significant that in this case the velocity remains equal to the initial one and the equations coincide in speed with the Lamb equation. In general, the influence of the moment is added. It follows that all the conclusions of the classical theory will be preserved.

\section{APPENDICES}

A new model of a continuous medium is proposed, based on taking into account the angular momentum through calculation outside the integral term using the Ostrogradsky-Gauss theorem. The model does not use the hypothesis of equal pressure of one third of the sum of pressures on the sides of the elementary volume parallel to the coordinate axes. The proof is the occurrence of a vortex sheet when solving flow problems according to the Euler model. The contribution of the moment becomes decisive in calculating the flow separation near the wings. The symmetry of the stress tensor is possible only if we ignore the additional term,which takes into account the rotation of the elementary volume, that creates additional force in the equation for the momentum.

\section{SOURCES OF FUNDING}

None. 


\section{CONFLICT OF INTEREST}

None.

\section{ACKNOWLEDGMENT}

None.

\section{REFERENCES}

[1] Loytsyansky. L.G Fluid Mechanics. M.: Nauka.1970. 904

[2] Kochin N.E., Kibel I.A., Rose N.V. Theoretical hydromechanics. Part 2. State publisher. physical and mathematical literature. 1963.727.

[3] LeBlond Paul H., Lawrence A. Mysak Lawrence A. Amsterdam, ...: Elsevier Waves in ocean. 1978.

[4] Katz A.M.. Theory of elasticity. St. Petersburg, Doe. 2002.208

[5] Prozorova. Evelina Influence of the dispersion in model of continuous mechanics. Lambert. 2018, 108

[6] Prozorova E. V. The Effect of Angular Momentum and Ostrogradsky-Gauss Theorem in the Equations of Mechanics. Wseas Transaction on fluid DOI: 10.37394/232013.2020.15.2

[7] Prozorova. ]. Evelina V. Effect of Mathematical Models on Experimental Data for the Gas and Liquids. Journal of Mechanics Engineering and Automation 6 (2016) 313-318 doi: 10.17265/2159-5275/2016.06.008 